\title{
Essais cliniques SMA en cours : diverses approches visant différentes cibles ${ }^{1}$
}

\author{
Tuy Nga Brignol
}

Parallèlement à l'autorisation de mise sur le marché du Spinraza ${ }^{\circledR}$ par la Food and Drug Administration (FDA) aux États-Unis en décembre dernier pour les formes adultes et pédiatriques de l'amyotrophie spinale (SMA), d'autres molécules sont en cours de développement. Voici ci-après un aperçu des essais en cours à travers le monde. Ils sont classés ici en fonction du mode d'action des molécules testées.

\section{Action sur SMN2}

Modification de l'épissage de l'ARN messager du gène SMN2 destinée à réintégrer l'exon 7 afin de produire une protéine SMN2 fonctionnelle en quantité suffisante.
1. Spinraza ${ }^{\oplus}$ (nusinersen ou ex-IONIS-SMNRx ou ex ISIS-SMNRx ou ex ISIS-396443 ou ex-BIIB058)

Oligonucléotide antisens développé par Ionis Pharmaceuticals Inc. en partenariat avec le laboratoire Biogen (pour sa commercialisation).

Voie d'administration : intrathécale.

\begin{tabular}{|c|c|c|c|c|c|c|c|c|}
\hline & Pays & Statut & $\begin{array}{c}\text { Nombre } \\
\text { de } \\
\text { patients }\end{array}$ & Phase & Âge & $\begin{array}{l}\text { Type } \\
\text { SMA }\end{array}$ & $\begin{array}{l}\text { Début- } \\
\text { Fin }\end{array}$ & $\begin{array}{l}\text { Durée } \\
\text { du suivi }\end{array}$ \\
\hline $\begin{array}{l}\text { Essai SHINE } \\
\text { NCT02594124 } \\
\end{array}$ & $\begin{array}{l}\text { France (I-Motion), Allemagne, } \\
\text { Australie, Belgique, Canada, } \\
\text { Espagne, États-Unis, } \\
\text { Grande-Bretagne, Hong Kong, } \\
\text { Italie, Japon, Corée, Suède, } \\
\text { Taïwan }\end{array}$ & $\begin{array}{l}\text { Recrutement } \\
\text { sur invitation }\end{array}$ & 289 & $\begin{array}{l}\text { III } \\
\text { (extension des } 4 \text { essais } \\
\text { ENDEAR, CHERISH, } \\
\text { NCT02052791 } \\
\text { NCT01839656 } \\
\text { sans placebo) }\end{array}$ & 13 mois à 21 ans & 1 et 2 & $\begin{array}{l}\text { Nov } 2015 \text { - } \\
\text { Août } 2022\end{array}$ & $\begin{array}{l}1807 \\
\text { jours }\end{array}$ \\
\hline $\begin{array}{l}\text { Essai EMBRACE } \\
\text { NCT02462759 } \\
\end{array}$ & États-Unis, Allemagne & $\begin{array}{l}\text { Recrutement } \\
\text { terminé } \\
\text { Essai en cours }\end{array}$ & 21 & $\begin{array}{l}\text { II } \\
\text { (avec placebo) }\end{array}$ & $\begin{array}{l}\text { Nourrissons, enfants, } \\
\text { adultes, seniors, (non } \\
\text { éligibles aux essais } \\
\text { ENDEAR et } \\
\text { CHERISH) }\end{array}$ & 1 à 4 & $\begin{array}{l}\text { Août } 2015 \text { - } \\
\text { Avril } 2019\end{array}$ & $\begin{array}{l}44 \\
\text { mois }\end{array}$ \\
\hline
\end{tabular}

Tuy Nga Brignol

AFM-Téléthon, Évry,

France

tnbrignol@afm-telethon.fr

\author{
NOTE \\ ${ }^{1}$ Source : \\ https://clinicaltrials.gov/ \\ (données au 15 avril \\ 2017).
}




\section{RO7034067 (RG7916)}

Molécule développée par le laboratoire Hoffmann-La Roche en partenariat avec PTC Therapeu-

tics.

Voie d'administration : orale.

\begin{tabular}{|c|c|c|c|c|c|c|c|c|}
\hline & Pays & Statut & $\begin{array}{c}\text { Nombre } \\
\text { de } \\
\text { patients }\end{array}$ & Phase & Âge & $\begin{array}{l}\text { Type } \\
\text { SMA }\end{array}$ & $\begin{array}{l}\text { Début- } \\
\text { Fin }\end{array}$ & $\begin{array}{l}\text { Durée } \\
\text { du suivi }\end{array}$ \\
\hline $\begin{array}{l}\text { Essai SUNFISH } \\
\text { NCT02908685 } \\
\end{array}$ & $\begin{array}{l}\text { France (Paris, Bron, Lille, } \\
\text { Nantes), Allemagne, Australie, } \\
\text { Belgique, Canada, Espagne, } \\
\text { États-Unis, Italie, } \\
\text { Royaume-Uni, Suède, Suisse, } \\
\text { Taïwan, Turquie }\end{array}$ & $\begin{array}{l}\text { Recrutement } \\
\text { en cours }\end{array}$ & 186 & $\begin{array}{l}\text { II } \\
\text { (avec placebo) }\end{array}$ & 2 à 25 ans & 2 et 3 & $\begin{array}{l}\text { Oct } 2016 \text { - } \\
\text { Mars } 2020\end{array}$ & $\begin{array}{l}\text { Partie } 1 \\
\text { (exploratoire) : } \\
12 \text { semaines } \\
\text { Partie } 2 \\
\text { (confirmatoire): } \\
24 \text { mois }\end{array}$ \\
\hline
\end{tabular}

\section{LMIO70}

Molécule développée par Novartis Pharmaceuticals.

Voie d'administration : orale.

\begin{tabular}{|c|c|c|c|c|c|c|c|c|}
\hline & Pays & Statut & $\begin{array}{c}\text { Nombre } \\
\text { de } \\
\text { patients }\end{array}$ & Phase & Âge & $\begin{array}{l}\text { Type } \\
\text { SMA }\end{array}$ & $\begin{array}{l}\text { Début- } \\
\text { Fin }\end{array}$ & $\begin{array}{l}\text { Durée } \\
\text { du suivi }\end{array}$ \\
\hline $\begin{array}{l}\text { Essai } \\
\text { NCT02268552 } \\
\end{array}$ & $\begin{array}{l}\text { Allemagne, Belgique, } \\
\text { Danemark, Italie }\end{array}$ & $\begin{array}{l}\text { Recrutement } \\
\text { en cours }\end{array}$ & 42 & $\begin{array}{l}\text { I/II } \\
\text { (sans placebo) } \\
\text { Essai en ouvert }\end{array}$ & 1 à 7 mois & 1 & $\begin{array}{l}\text { Auril } 2015 \text { - } \\
\text { Août } 2017\end{array}$ & 13 semaines \\
\hline
\end{tabular}

\section{Apport du gène SMN}

AVXS-101

Molécule développée par AveXis.

Suite aux résultats intermédiaires encourageants de

l'essai en cours, le laboratoire prépare, courant 2017, un nouvel essai dans la SMA de type 1 aux
États-Unis puis en Europe, et un essai dans la SMA de type 2.

Voie d'administration: une administration unique par cathéter veineux inséré dans une veine périphérique.

\begin{tabular}{|c|c|c|c|c|c|c|c|c|}
\hline & Pays & Statut & $\begin{array}{c}\text { Nombre } \\
\text { de } \\
\text { patients }\end{array}$ & Phase & Âge & $\begin{array}{l}\text { Type } \\
\text { SMA }\end{array}$ & $\begin{array}{l}\text { Début- } \\
\text { Fin }\end{array}$ & $\begin{array}{l}\text { Durée } \\
\text { du suivi }\end{array}$ \\
\hline $\begin{array}{l}\text { Essai } \\
\text { AVXS-101 } \\
\text { NTC02122952 }\end{array}$ & États-Unis & $\begin{array}{l}\text { Recrutement } \\
\text { terminé, } \\
\text { essai en cours }\end{array}$ & 15 & $\begin{array}{l}\text { I } \\
\text { (sans placebo) }\end{array}$ & $\leq 6$ mois & 1 & $\begin{array}{l}\text { Auril } 2014 \text { - } \\
\text { Déc } 2017\end{array}$ & 2 ans \\
\hline
\end{tabular}




\section{Protection du motoneurone et/ou du muscle}

\section{Olesoxime}

Molécule visant à protéger le motoneurone, mise au point par Trophos avec le soutien de l'AFM-
Téléthon, rachetée et développée par le laboratoire Hoffmann-La Roche.

Voie d'administration: orale, sous forme de sirop, ou par sonde gastrique.

\begin{tabular}{|c|c|c|c|c|c|c|c|c|}
\hline & Pays & Statut & $\begin{array}{c}\text { Nombre } \\
\text { de patients }\end{array}$ & Phase & Âge & $\begin{array}{l}\text { Type } \\
\text { SMA }\end{array}$ & $\begin{array}{l}\text { Début- } \\
\text { Fin }\end{array}$ & $\begin{array}{l}\text { Durée } \\
\text { du suivi }\end{array}$ \\
\hline $\begin{array}{l}\text { Essai } \\
\text { NTC02628743 } \\
\end{array}$ & $\begin{array}{l}\text { France (Bron, Garches, Lille, } \\
\text { Marseille, Montpellier, Paris, } \\
\text { Toulouse), Allemagne, } \\
\text { Belgique, Italie, Pays-Bas, } \\
\text { Pologne, Royaume-Uni }\end{array}$ & $\begin{array}{l}\text { Recrutement } \\
\text { terminé, } \\
\text { essai en cours }\end{array}$ & $\begin{array}{l}\text { Patients } \\
\text { ayant participé } \\
\text { aux essais } \\
\text { précédents } \\
\text { de phases Ib et II }\end{array}$ & $\begin{array}{l}\text { Extension } \\
\text { de la phase II }\end{array}$ & $\begin{array}{l}\text { Enfants, adultes, } \\
\text { seniors }\end{array}$ & $2,3,4$ & $\begin{array}{l}\text { Janv } 2016 \text { - } \\
\text { Déc } 2020\end{array}$ & 4 ans \\
\hline
\end{tabular}

\section{CK-2127107}

Molécule développée par Cytokinetics en collaboration avec Astellas Pharma Global Development Inc pour améliorer la fonction musculaire.

Voie d'administration : orale.

\begin{tabular}{|l|l|l|l|l|l|l|l|l|}
\hline & \multicolumn{1}{|c|}{ Pays } & \multicolumn{1}{|c|}{ Statut } & $\begin{array}{l}\text { Nombre } \\
\text { de } \\
\text { patients }\end{array}$ & \multicolumn{1}{|c|}{ Phase } & Âge & $\begin{array}{c}\text { Type } \\
\text { SMA }\end{array}$ & $\begin{array}{c}\text { Début- } \\
\text { Fin }\end{array}$ & $\begin{array}{c}\text { Durée } \\
\text { du suivi }\end{array}$ \\
\hline $\begin{array}{l}\text { Essai CY 5021 } \\
\text { NTC02644668 }\end{array}$ & États-Unis, Canada & $\begin{array}{l}\text { Recrutement } \\
\text { terminé, } \\
\text { essai en cours }\end{array}$ & 72 & $\begin{array}{l}\text { phase II } \\
\text { (avec placebo) }\end{array}$ & $\geq 12$ ans & $2,3,4$ & $\begin{array}{l}\text { Déc 2015- } \\
\text { Juil 2017 }\end{array}$ & 12 semaines \\
\hline
\end{tabular}

\section{Pyridostigmine (mestinon)}

Pour améliorer la fatigabilité musculaire en préser-

vant la jonction neuromusculaire.

Voie d'administration : orale.

\begin{tabular}{|c|c|c|c|c|c|c|c|c|}
\hline & Pays & Statut & $\begin{array}{c}\text { Nombre } \\
\text { de } \\
\text { patients }\end{array}$ & Phase & Âge & $\begin{array}{l}\text { Type } \\
\text { SMA }\end{array}$ & $\begin{array}{l}\text { Début- } \\
\text { Fin }\end{array}$ & $\begin{array}{l}\text { Durée } \\
\text { du suivi }\end{array}$ \\
\hline $\begin{array}{l}\text { Essai } \\
\text { SPACE } \\
\text { NTC02941328 } \\
\end{array}$ & Pays-Bas & $\begin{array}{l}\text { Recrutement } \\
\text { en cours }\end{array}$ & 50 & $\begin{array}{l}\text { II } \\
\text { (avec placebo) }\end{array}$ & $\geq 12$ ans & $2,3,4$ & $\begin{array}{l}\text { Déc } 2015 \text { - } \\
\text { Déc } 2017\end{array}$ & 16 semaines \\
\hline $\begin{array}{l}\text { Essai } \\
\text { EMOTAS } \\
\text { NTC02227823 }\end{array}$ & Belgique & $\begin{array}{l}\text { Recrutement } \\
\text { en cours }\end{array}$ & 20 & $\begin{array}{l}\text { II } \\
\text { (avec placebo) }\end{array}$ & $\geq 6$ ans & 3 & $\begin{array}{l}\text { Juil } 2014 \text { - } \\
\text { Juil } 2017\end{array}$ & 6 mois \\
\hline
\end{tabular}

\section{LIENS D'INTÉR̂̂T}

L'auteur déclare n'avoir aucun lien d'intérêt concernant les données publiées dans cet article.

Ongoing clinical trials in SMA: distinct targets based on distinct approaches 